\title{
EXTENDING IMAGE RETRIEVAL WITH GROUP-ORIENTED INTERFACE
}

\author{
Munehiro Nakazato and Thomas S. Huang \\ Beckman Institute for Advanced Science and Technology \\ University of Illinois at Urbana-Champaign, Urbana, IL 61801, USA \\ \{nakazato, huang\}@ifp.uiuc.edu
}

\begin{abstract}
ImageGrouper is a new user interface for digital image search and organization. It is group-oriented in that image search, annotation and organizing are done by grouping images on the workspace. This enables faster and flexible text annotation on a large number of images as well as effective image organization. In addition, this interface improves and extends Content-Based Image Retrieval (CBIR) as well. First, finding good combinations of query examples is essential for successful retrieval. Query-byGroups concept allows the user to try different combinations of query examples quickly and easily. Second, because of semantic gap between the user's concept and extracted visual features, it may be difficult to represent the user's request by only one class of query examples. We propose a new feature-weighting scheme where the user's request can be expressed in multiple positive classes.
\end{abstract}

\section{INTRODUCTION}

In Content-based Image Retrieval (CBIR) [3][6][7][11], Query-by-Example is commonly used. In this method, the users select example images (as positive or negative) and ask the system to retrieve visually similar images. The similarity between images is computed based on extracted visual features such as color [10] and texture [9]. In addition, in order to improve the retrieval, CBIR systems often employ relevance feedback [6][11], in which the users refine the search incrementally by giving feedback to the result of the previous query.

We are developing a new tool for digital image search and organization, named ImageGrouper [5]. ImageGrouper is a group-oriented interface in that every operation is based on group configurations. The users search, annotate an organize images by creating groups of images. In this paper, we describe how the design of our new system addresses problems in the traditional image retrieval systems. We propose a new feature-weighting scheme that can handle the user's request as multiple positive and multiple negative classes problems.
In the next section, the basic concepts of ImageGrouper are briefly introduced. In Section 3 and Section 4 , we describe how our system improves and extends content-based image retrieval.

\section{THE IMAGEGROUPER}

In this section, we briefly introduce the basic concepts of ImageGrouper: Query-by-Groups, Annotate-by-Groups and Organize-by-Groups.

\subsection{Query-by-Groups}

In ImageGrouper, a new concept Query-by-Groups is introduced to content-based image retrieval. This is an extension to Query-by-Example method that is commonly used in image retrieval [6][11]. The major difference is that while Query-by-Example handles the images individually, a group of images is considered as the basic unit of the query in Query-by-Group.

Figure 1. shows interface of the ImageGrouper. The display is divided into two panes. The left pane is the ResultView that displays the results of both content-based and keyword-based search. The right pane is the GroupPalette, in which the user manipulates image groups directly. In order to create an image group, the user first drags images from the ResultView to GroupPalette, then

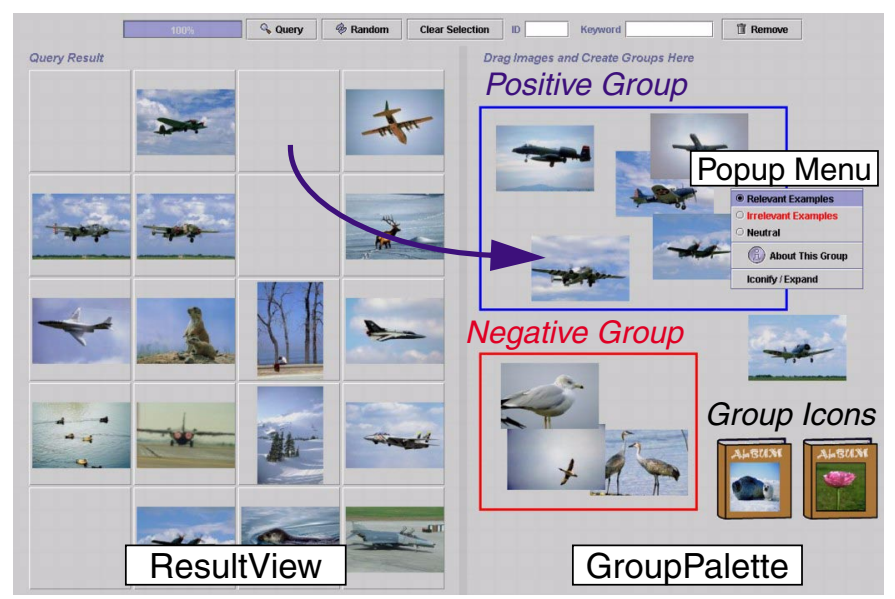

Figure 1. ImageGrouper Interface (annotated) 
encloses the images by drawing a box as we draw rectangles in an illustrating application. The user can create any number of groups within the palette. For each group, the user can specify it as relevant, irrelevant or neutral. Neutral groups do not contribute to the search at the moment and can be used for another query later. The properties of the groups can be easily changed from the popup menu. The box colors change accordingly. Moreover, the user can move images from one group to another at any moment. In addition, these groups can be overlapped to each other, allowing images to belong multiple groups.

When the user presses the "Query" button, the system retrieves images that are similar to positive group images while avoiding images similar to negative group images. The results are displayed in the ResultView. The user can refine the query further by dragging the new images into groups in the palette.

\subsection{Annotating and Organizing Images by Groups}

The concept of "groups" is also used for text annotation and image organization.

No wonder keyword-based image search is a very powerful method. The problem is that it works only when all images are annotated with textual information. Annotating a large number of images is, however, a tedious task. ImageGrouper introduces Annotation-byGroups method where keywords are annotated not on individual images, but on groups. Because the users can simultaneously annotate the same keywords on a number of images, annotation becomes much faster and less error prone. Furthermore, when groups are overlapped, images in the overlapped region are associated with keywords of both groups. By creating a group within an existing group, hierarchical annotation is possible.

Once images are annotated, they can be used for keyword-based search. In ImageGrouper, keyword-based search and content-based search are closely related and complementary to each other. Content-based retrieval by Query-by-Groups is used to collect images to annotate. Conversely, keyword-based search can be used to find a stating point for content-based retrieval.

Meanwhile, these groups can be used to organize pictures as "photo albums [4]" When the user points a group and choose "Iconfify" from the popup menu, images in the group are shrunk into Album icons (shown in the lower right of Figure 1.) These icons can be moved anywhere in the GroupPalette. The user can expand an icon back into an image group where images are surrounded by a rectangle. In addition, album icons themselves can be used as query example for additional query. To this end, the user selects query property (positive, negative, neutral) of the icon from the popup menu. Based on this property, the color of the album changes.

In the following sections, we describe how ImageGrouper improves and extends content-based image retrieval.

\section{SUPPORTING FAST EXPERIMENTAL QUERY}

ImageGrouper addresses several drawbacks in traditional user interfaces of CBIR systems that employ Query-byExample. First, the traditional CBIR systems assume that the more query examples are available, the better result we can get. Therefore, the users are supposed to search images only by adding new example images incrementally from the previous query results. When no additional example is found in the result set, the search is considered to have converged. However, this assumption is not necessarily true. There are cases where additional examples may contain undesired features and degenerate the retrieval performance. In [5], Nakazato et al. showed that finding good combinations of query examples is essential for successful image retrieval. Unfortunately, this task was difficult or inefficient in the traditional interfaces.

Second, the traditional systems do not allow the user to put aside the query results for later uses. This type of interaction is desired because the users of CBIR are not necessarily looking for only one type of images. The users' interest may change during retrieval. This behavior is known as berry picking and have been observed for text document retrieval [1]. Moreover, because of semantic gap [8] between the high-level concept in the user's mind and the extracted low-level image features, the uses often need to make more than one query to satisfy their need. Therefore, support for trial-and-error query (or experimental query) is desirable.

Compared with the traditional GUI, ImageGrouper is much easier to try different combinations of query examples. Images can easily enter or leave a group by mouse drags. Thus, the user can quickly explore different combinations of query examples by dragging images into or out of the box on the GroupPalette. It is shown that with the trial-and-error query, retrieval performance is improved even if relevance feedback algorithm is identical [5]. Therefore, ImageGrouper can achieve better retrieval (higher recall) without any modification to relevance feedback algorithm. In the next section, however, we will extend relevance feedback algorithm itself so that we can further take advantage of Query-by-Group interaction.

Moreover, the system provides storage area for images that are not used for query at the moment. They can be used for another query later. In addition, the user can create new groups within an existing group (Group in a group). Thus, the user can begin with collecting relatively generic images first, then narrow down the 
search to more specific images. For example, assume the user is looking for "red car" images. S/he can initially collect "cars with any colors." Once s/he found enough number of images, s/he can divide them into two groups: red cars and other cars. Then the user can specify red car group as relevant, and other cars as irrelevant. This narrowing down search was not possible with the conventional interfaces.

\section{EXTENDING FEATURE DISTANCE METRIC}

In interactive content-based image retrieval, the system needs to re-calculate distance metric (or feature weighting) based on the user's feedback (relevance feedback [7][11].) This problem is reduced into online calculation of feature space transformation matrix.

\subsection{Related Work}

\subsubsection{Traditional approaches}

When negative image examples are not considered, the simplest way is to calculate the variance of each feature among the query examples. Then, the inverse of variance becomes the weight of the feature [6]. This gives higher weights to features in which example images are similar. If the number of example images is high, it is known that the whitening transform is the optimal choice [7].

Next, when negative examples are introduced, the problems can be addressed as two-class (positive and negative) problems. One solution is to use the Fisher's discriminant analysis (FDA) [11]. This approach, however, introduces undesirable side effects because it tries to cluster negative examples into one class. In the actual scenario, negative examples can be any types of images in the database other than the positive class.

\subsubsection{Image retrieval as a $(1+x)$-class problem}

Zhou et al.[11] proposed a new relevance feedback algorithm, which effectively takes negative examples into account. They consider the relevance feedback problem as $(1+x)$-class (one positive and multiple negative classes) problem. In this scheme, it is assumed the negative examples are coming from an uncertain number of classes, while the positive can be clustered into one class. Their algorithm, named Biased Discriminant Analysis (BDA) is characterized by the following objective function,

$$
\boldsymbol{W}=\underset{\boldsymbol{W}}{\operatorname{argmax}}\left|\frac{\boldsymbol{W}^{T} \boldsymbol{S}_{\text {bias }} \boldsymbol{W}}{\boldsymbol{W}^{T} \boldsymbol{S}_{W} \boldsymbol{W}}\right|
$$

where,

$$
\begin{gathered}
\boldsymbol{S}_{W}=\sum_{x \in C}(\boldsymbol{x}-\boldsymbol{m})(\boldsymbol{x}-\boldsymbol{m})^{T} \\
\boldsymbol{S}_{\text {bias }}=\sum_{y \in D}(\boldsymbol{x}-\boldsymbol{m})(\boldsymbol{x}-\boldsymbol{m})^{T}
\end{gathered}
$$

$C$ is a set of positive examples and $D$ is a set of negative examples. In short, BDA tries to minimize within-class scatter matrix $\boldsymbol{S}_{W}$ of the positive example, while $\boldsymbol{S}_{\text {bias }}$ is

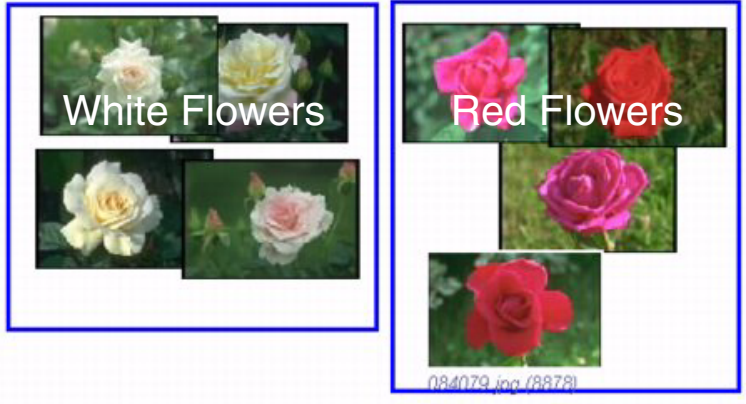

Figure 2. White flowers and Red flowers. In ImageGrouper, users can separate them into two positive groups.

keeping the negative examples away from the positive examples.

\subsection{Extending to $(x+y)$-class problem}

In the first version [5] of ImageGrouper, when the user specifies more than one groups as positive, these groups are merged into one group for query. Thus, biased discriminant analysis described above was used, i.e. one positive group and multiple negative groups.

However, this approach is not taking full advantages of our group-oriented interface. When the user searches images, s/he may have high-level concept in her/his mind such as "beautiful flowers." Such concept cannot be expressed by only one class of images. See Figure 2. Although white flowers and red flowers may have common visual features, they also have very different features, which is the color. If the system tries to consider them as one class of images, the color features have to be discarded. This is not desirable because these features may be beneficial to retrieve a specific color of flowers.

Even for the users, it is more intuitive if they can specify them as two different, but closely related positive classes. This fact motivated us to extend the relevance feedback problems to $(x+y)$-class (multiple positive and multiple negative classes) problem.

In our new scheme, the objective function of the new approach becomes as follows,

$$
\boldsymbol{W}=\underset{\boldsymbol{W}}{\operatorname{argmax}}\left|\frac{\boldsymbol{W}^{T} \boldsymbol{S}_{P N} \boldsymbol{W}}{\boldsymbol{W}^{T} \boldsymbol{S}_{W} \boldsymbol{W}}\right|
$$

where $\boldsymbol{S}_{W}$ is the sum of the within-class scatter matrix [2] of the positive groups defined as follows,

$$
\begin{gathered}
\boldsymbol{S}_{W}=\sum_{i=1}^{c} \boldsymbol{S}_{i} \\
\boldsymbol{S}_{i}=\sum_{x \in C_{i}}\left(\boldsymbol{x}-\boldsymbol{m}_{i}\right)\left(\boldsymbol{x}-\boldsymbol{m}_{i}\right)^{T}
\end{gathered}
$$

$\boldsymbol{m}_{i}$ is the mean vector of $i$-th positive class $C_{i}, c$ is the number of positive groups. $S_{P N}$ is the positive-to-negative scatter, which is introduced in this paper as follows,

$$
\boldsymbol{S}_{P N}=\sum_{i=1}^{c} \boldsymbol{S}_{N i}
$$




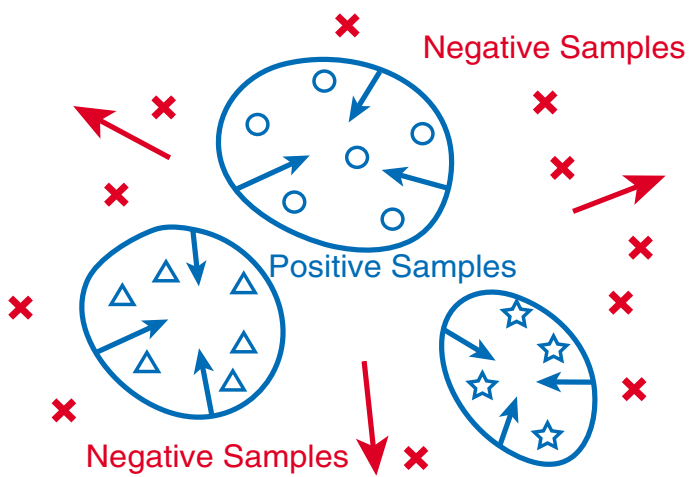

Figure 3. Concept of the new feature space transform. It minimizes the scatter of each positive class while maximizing the scatter between positive and negative samples.

$$
\boldsymbol{S}_{N i}=\sum_{y \in D}\left(\boldsymbol{y}-\boldsymbol{m}_{i}\right)\left(\boldsymbol{y}-\boldsymbol{m}_{i}\right)^{T}
$$

where, $D$ is a set of negative examples.

Figure 3. visualizes the concept of our scheme. In short, this scheme tries to cluster each positive class while scatter the negative example away from the positive classes.

As in FDA and BDA described above, $\boldsymbol{W}$ is solved as the generalized eigenvector(s) associated with the largest eigenvalue(s) $\lambda$,

$$
\boldsymbol{S}_{P N} \boldsymbol{w}_{i}=\lambda_{i} \boldsymbol{S}_{W} \boldsymbol{w}_{i}
$$

Finally, our discriminating transformation matrix [2] becomes,

$$
\boldsymbol{A}=\Phi \Lambda^{1 / 2}
$$

$\Phi$ is the matrix whose columns are the eigenvectors, and $\Lambda$ is the diagonal matrix of the corresponding eigenvalues. Once the transformation matrix is available, the distance between two images $\boldsymbol{x}$ and $\boldsymbol{y}$ is computed as follows.

$$
\operatorname{dist}(\boldsymbol{x}, \boldsymbol{y})=(\boldsymbol{x}-\boldsymbol{y})^{T} \boldsymbol{A}(\boldsymbol{x}-\boldsymbol{y})
$$

In our current implementation, we compare the distance between images in the database and the mean of each positive group. Then, the database images are ordered according to those distances.

\section{IMPLEMENTATION}

ImageGrouper is implemented as a client-server system. The interface client is implemented as a Java2 applet so that the users can use the system through web browsers on various platforms. Computation of discriminating transformation matrix is done on the server side. It is implemented as a Java Servlet, which is written in Java and $\mathrm{C}++$ with MATLAB C++ Math Library.

\section{CONCLUSION}

In this paper, we described how our new user interface ImageGrouper improves and extends relevance feedback for content-based image retrieval. First, the system supports effective experimental query. The user can quickly try different combinations of query examples by dragging image on the workspace.

In addition, we introduced a new feature weighting scheme. In this method, the user's interest can be expressed as $(x+y)$-class (multiple positive and multiple negative) problems. Our new user interface inspired this new scheme. In fact, there was no way to apply this scheme on the traditional interfaces because the user could not specify positive example as multiple classes.

Readers can try ImageGrouper demo online at http://www. ifp.uiuc. edu/ nakazato/grouper/

\section{ACKNOWLEDGEMENT}

This work was supported in part by National Science Foundation Grant CDA 96-24396.

\section{REFERENCES}

[1] Bates, M.J. The design of browsing and berrypicking techniques for the on-line search interface. Online Review, 13(5), pp. 407-431, 1989.

[2] Duda, R.O., Hart, P.E. and D.G. Stork, Pattern Classification (2nd Ed.), Wiley-Interscience, 2000.

[3] Flickner, M. et al., "Query by image and video content: The QBIC system," IEEE Computers, 1995.

[4] Kuchinsky,A. et al. FotoFile: A Consumer Multimedia Organization and Retrieval System. In CHI'99.

[5] Nakazato, M., Manola, L. and Huang, T.S., "ImageGrouper: Search, Annotate and Organize Images by Groups," In Proc. of 5th Intl. Conf. on Visual Information Systems (VIS'02), 2002.

[6] Rui, Y., et al., "Relevance Feedback: A Power Tool for Interactive Content-Based Image Retrieval," In IEEE Trans. on Circuits and Video Technology, Vol.8, No.5, Sept. 1998

[7] Rui, Y. and Huang, T. S., "Optimizing Learning in Image Retrieval," In Proc. of IEEE CVPR, 2000.

[8] Santini, S. and Jain, R., "Integrated Browsing and Querying for Image Database," IEEE Multimedia, Vol. 7, No. 3, 2000, page 26-39.

[9] Smith, J.R. and Chang S-F. Transform features for texture classification and discrimination in large image databases. In Proc. of IEEE International Conference on Image Processing, 1994.

[10] Sticker, M. and Orengo, M., Similarity of Color Images. In Proc. of SPIE, Vol. 2420 (Storage and Retrieval of Image and Video Databases III), 1995.

[11]Zhou, X. S., Petrovic, N. and Huang, T. S. Comparing Discriminating Transformations and SVM for Learning during Multimedia Retrieval. In Proceedings of ACM Multimedia '01, 2001. 